\title{
Los gestos deícticos y su impacto en el lenguaje productivo de infantes
}

Aline Minto García, Elda Alicia Alva Canto,

Natalia Arias Trejo

\section{Resumen}

Los gestos de las madres influyen en el desarrollo del vocabulario de sus hijos (Iverson et al., 1999). El objetivo de este estudio es analizar la relación entre la producción gestual de las madres y la producción léxica de sus hijos en una tarea de lectura conjunta. Participaron 30 díadas madre-infante, monolingües hablantes nativos del español: 15 con infantes de 30 meses y 15 con infantes de 48 meses, niños y niñas con desarrollo típico. Se realizó una tarea de lectura conjunta; se registró la producción de gestos deícticos de las madres y la producción léxica de los infantes (palabras type y token). En las díadas de infantes de 48 meses, se encontró una correlación positiva entre la producción gestual de las madres y la amplitud en la producción de palabras de sus hijos. La gestualidad de las madres es una variable asociada al tamaño del vocabulario productivo de sus hijos.

Palabras clave: gestos; vocabulario productivo; lectura conjunta.

La presente investigación fue realizada gracias al apoyo del Programa de Becas Posdoctorales DGAPA-UNAM periodo 2017-2018, asignada a la Dra. Aline Minto García. 


\section{Introducción}

El desarrollo del lenguaje verbal de los infantes ha sido asociado principalmente a los estímulos orales que reciben de sus padres (Pan, Rowe, Singer y Snow, 2005); sin embargo, el habla emerge acompañada de otros recursos comunicativos como los gestos que refuerzan o agregan información a los mensajes orales (Özçalişkan y Goldin-Meadow, 2005). Desde una perspectiva psicolingüística, los gestos son movimientos de las manos o brazos que las personas realizan mientras hablan. Los gestos precisan información junto con las palabras y son elementos constitutivos de la significación (McNeill, 1992); en este sentido, los gestos poseen un vínculo estrecho con el discurso y juegan un papel relevante en el esquema comunicativo (Bavelas, Kenwood, Johnson y Phillips, 2002).

Los gestos, a diferencia de las simples acciones llevadas a cabo con las manos -cuya finalidad es cumplir con una función práctica en el mundo material-, tienen una función comunicativa en el mundo social (Minto-García, 2014); implican actos simbólicos (Bavelas y Chovil, 2006). Los movimientos gestuales representan conceptos de la mente (Kendon, 2007) que no siempre son o pueden ser expresados a través de las palabras (Bavelas et al., 2002; De Ruiter, Bangerter y Dings, 2012; Goldin-Meadow, 2000; Özçalişkan, Genter y Goldin-Meadow, 2014).

McNeill (1992) ofrece una categorización de los gestos según la relación que establecen con el referente: deícticos, icónicos, metafóricos y rítmicos. El presente estudio se centró en los gestos deícticos dado que son los más usados en las interacciones madre e hijo (Iverson, Capirci, Longobardi y Caselli, 1999). Los gestos deícticos son movimientos que apuntan o señalan objetos; típicamente estos gestos son realizados con los dedos -por ejemplo, con el dedo índice extendido-, aunque puede ser usado cualquier objeto extensible o alguna otra parte del cuerpo -la cabeza, la nariz, el mentón (Haviland, 2000; Krauss, Chen y Gottesman, 2000; McNeill, 1992). Aunque básicamente su función es referencial, a través de ellos también se puede mostrar, ofrecer y dar un objeto, así como realizar una petición a manera de ritual (Farkas, 2007). 
Algunas investigaciones han mostrado que los gestos favorecen el desarrollo cognitivo de los infantes (Goldin-Meadow, 2000); por ejemplo, benefician los procesos de comprensión y aprendizaje de palabras (Capone y Saks, 2015; Cook y Goldin-Meadow, 2006). Ahora bien, la producción de gestos en la infancia también ha sido asociada al desarrollo del lenguaje verbal de los infantes; se ha observado que los gestos que producen los niños predicen la aparición de hitos lingüísticos (Capone y McGregor, 2004; Goldin-Meadow, 2015; Kuhn et al., 2014); y que los gestos producidos por los padres tienen un impacto en el vocabulario de los infantes (Iverson et al., 1999).

Es ampliamente conocido que los padres gestualizan cuando se comunican con sus hijos (Acredolo y Goodwyn, 1988; Özçalişkan y Dimitrova, 2013). Existe evidencia sobre cómo los gestos suelen reforzar el mensaje o bien transmitir información que no se expresa por medios orales (Greenfield y Smith, 1976; Özçalişkan y Goldin-Meadow, 2005; Zukow-Goldring, 1996). En este sentido, es altamente probable que la gestualidad de los padres pueda facilitar la comprensión de un mensaje dirigido a sus hijos (Rowe, Özçalişkan y Goldin-Meadow, 2008; Stanfield, Williamson y Özçalişkan, 2013).

Según Iverson et al. (1999), los gestos de las madres influyen en el desarrollo posterior del vocabulario de sus hijos. Después de observar interacciones lúdicas y momentos de comida entre madres e hijos italianos, estos autores identificaron una alta correlación positiva entre la producción de gestos deícticos de la madre y el tamaño del vocabulario de sus hijos a los 16 meses de edad. Asimismo, reportaron que la producción gestual que las madres realizan cuando sus hijos poseen 16 meses de edad predice la producción de palabras de los infantes a los 20 meses.

En una investigación cuyo objetivo fue evaluar si el uso del gesto marca la diferencia en el desarrollo del vocabulario entre niños de nivel socioeconómico alto y bajo, se encontró que el uso temprano del gesto por parte de los padres está asociado a las diferencias en el tamaño del vocabulario (Rowe y Goldin-Meadow, 2009). Este estudio identificó que los padres de nivel socioeconómico alto usan más gestos para comunicarse con sus 
hijos -y que estos gestos permiten que lo hagan con una gama más amplia de significado-; esto trae consigo que esos infantes, a su vez, usen una mayor cantidad de gestos a los 14 meses de edad -a diferencia de los infantes de nivel socioeconómico bajo. Esta diferencia en el uso de gestos en los infantes está asociada con su tamaño del vocabulario en el momento de ingresar a la escuela (a los 54 meses de edad). Con estos resultados se puede inferir que la gestualidad de los padres es un mecanismo que podría ser determinante en el tamaño del vocabulario de sus hijos.

En otro estudio, Rowe, Özçalişkan y Goldin-Meadow (2008) exploraron si la producción gestual de los padres se relaciona con el desarrollo posterior del vocabulario de sus hijos. A pesar de que no pudieron probar que la producción del gesto de los padres (a los 14 meses de edad de sus hijos) se relaciona directamente con el desarrollo del vocabulario del infante, encontraron que dicha relación se presenta de forma indirecta: la producción gestual de los infantes a los 14 meses, asociada a la producción gestual de sus padres, es un predictor de su vocabulario a los 42 meses. Los resultados de estas investigaciones indican la relevancia de analizar el uso del gesto de los padres como posible mecanismo de influencia en el desarrollo verbal de los niños.

Con el afán de encontrar evidencia sobre la relevancia de la producción gestual de las madres, surge la pregunta de investigación del presente estudio: ¿Los gestos deícticos de las madres tienen impacto en la producción léxica de sus hijos en un mismo intercambio comunicativo? Partiendo de lo anterior, el objetivo es analizar la relación entre la producción gestual de las madres y la producción léxica de sus hijos en una tarea de lectura conjunta. Cabe señalar que la lectura conjunta entre madres e hijos permite observar la riqueza de las estrategias comunicativas que los hablantes ponen en marcha (Arias, 2014). Se ha probado que en este tipo de actividades las madres utilizan un vocabulario más amplio y propician la interacción con sus hijos mediante la frecuente formulación de preguntas y usan con menor frecuencia el discurso regulador o directivo (Jones y Adamson, 1987; Lewis y Gregory, 1987). 
Se hipotetizó una correlación entre la producción de gestos deícticos de la madre y la producción de palabras de sus hijos. Así, entre más gestos produzca la madre en la tarea de lectura conjunta, especialmente en el momento en que cuenta su relato, mayor será la producción de palabras de los infantes en esta misma interacción comunicativa.

\section{Método}

\section{Participantes}

Participaron 30 díadas madre-infante, monolingües hablantes nativos del español: 15 con infantes de 30 meses $(M=29.9, D E$ $=0.47)$ y 15 con infantes de 48 meses $(M=48.3, D E=0.55)$, niños y niñas con desarrollo típico. Los criterios de inclusión para los infantes fueron: nacidos a término y sin problemas prenatales, perinatales o posnatales, así como la ausencia de problemas de audición o visión y de antecedentes de enfermedades graves. Respecto a las madres participantes de este estudio, poseen una media de edad de 33.67 años $(D E=6.87)$ y una media de escolaridad de 15.87 años de estudio ( $D E=1.76)$.

\section{Instrumentos}

Se empleó un cuestionario sociodemográfico con la finalidad de recabar datos generales de los participantes: fecha de nacimiento del niño, condiciones de nacimiento y antecedentes de enfermedades, datos relevantes de la madre, por mencionar algunos. Este instrumento arrojó información que permitió aplicar los criterios de inclusión.

Para la tarea de lectura conjunta, se empleó un libro ilustrado según la edad de los infantes: $A$ boy, a dog, and a frog, dirigido a infantes de 30 meses, y Frog on his own, enfocado a infantes de 48 meses (Mayer, 1967 y 1973). Estos libros presentan una serie de 24 ilustraciones sin texto. Estos materiales han mostrado ser instrumentos pertinentes para estudiar el lenguaje productivo -desde el nivel léxico hasta el nivel narrativo- (Romero y Gómez, 2013; Suárez, Alva y Valdés, 2016), ya que proveen al 
infante recursos -imágenes de escenas relacionadas temporal y causalmente- que fomentan su producción verbal; asimismo, han sido empleados en investigaciones enfocadas en el estudio de los gestos y el lenguaje (Kuhn et al., 2016; O'Neill y Holmes, 2002).

Para la videograbación de las sesiones de lectura conjunta, se utilizó una cámara digital Canon Vixia HF M40. Esta cámara se instaló en el cuarto de laboratorio -donde se realizó la lectura conjunta- momentos antes de la llegada de los participantes; se colocó en un soporte fijo a la pared (a una altura de $1.60 \mathrm{~m}$ del piso y a $1.90 \mathrm{~m}$ de distancia de la díada) frente a los participantes.

Recolección de los datos

Las madres fueron informadas sobre la tarea que realizarían con sus hijos y firmaron un consentimiento informado a través del cual otorgaron su permiso para que ambos participantes fueran grabados. En un primer momento, las madres contestaron un cuestionario sociodemográfico.

La sesión de lectura conjunta se realizó en un cuarto de laboratorio con las condiciones propicias para realizar la actividad: buena iluminación, espacio suficiente para el movimiento y asientos cómodos. Las instrucciones fueron las siguientes: "Le proporcionaremos un libro ilustrado que no contiene palabras. Por favor narre a su hijo una historia con base en los dibujos del libro y, cuando haya terminado de contarle la historia, entréguele el libro y pídale que ahora él cuente una historia a usted. Cuando terminen de contar ambos relatos, por favor abra la puerta del cubículo en señal de que han terminado". Así, sin la presencia del investigador, los participantes llevaron a cabo la tarea de lectura conjunta cuya duración aproximada fue de 20 minutos; todas las sesiones fueron videograbadas.

Codificación y análisis de los datos

La codificación de los gestos deícticos se realizó con base en los planteamientos de McNeill (1992). Aunque el conjunto de gestos deícticos incluye cuatro tipos -deícticos de pedir, dar, mostrar y 
señalar un objeto (Capirci, Iverson, Pizzuto y Volterra, 1996)-, en este estudio únicamente se tomó en cuenta el gesto deíctico de señalar. Así, se codificó como gesto deíctico a todo movimiento de manos o dedos que señaló o indicó un referente físico o imaginario. El estudio se limitó a este tipo de gestos porque tienen una alta prevalencia en las interacciones entre madre e hijo y han sido asociados al desarrollo del lenguaje (Iverson et al., 1999).

Las grabaciones de las sesiones de lectura conjunta fueron segmentadas y analizadas en lapsos de 10 segundos para identificar la cantidad de gestos deícticos producidos por las madres en el momento en que narraban su historia. Este tratamiento analítico temporal es uno de los requisitos procedimentales del método observacional, ya que asegura datos más exactos entre más cortas sean las unidades temporales; normalmente, las medidas se codifican en segmentos de 5 a 10 segundos, lo cual permite reducir el esfuerzo analítico (Anguera, 1983; Guerrero, 2016). En este estudio, dicha segmentación permitió una identificación más precisa de los gestos.

Respecto a la producción léxica de los infantes, primero, se realizó la transcripción completa de las grabaciones para una posterior revisión y confiabilización de los datos. La producción léxica fue analizada a partir de las medidas type y token -para realizar esta medición fue necesario realizar una codificación con base en nueve categorías gramaticales (Alva, 2004). La medida type corresponde a todas las palabras diferentes entre sí, que juntas conforman la amplitud del vocabulario; mientras que la medida token se refiere a todas las palabras emitidas por un individuo, que juntas conforman el vocabulario de redundancia. Cuando los investigadores tuvieron dificultades para identificar o transcribir una palabra -o fragmento-, se codificó como ininteligible.

El examen de los datos estuvo centrado en el análisis de la correlación entre el número de gestos deícticos producidos por la madre y el número de palabras (type-token) producidas por los infantes. Para ello se contabilizó la cantidad de gestos deícticos producidos por las madres, así como el total de palabras type y el total de palabras token. Se utilizó la prueba de correlación de Pearson para el análisis de los datos. 


\section{Resultados}

El objetivo de esta investigación fue analizar la relación entre los gestos deícticos de las madres y la producción de palabras de sus hijos en una tarea de lectura conjunta. Respecto a las madres que conformaron díadas con hijos de 30 meses de edad, se identificó una media de producción de gestos deícticos de 55.40 $(D E=21.39)$. Por su parte, las madres de los infantes de 48 meses obtuvieron una media de producción de gestos deícticos de $48.60(D E=26.22)$. No se encontraron diferencias significativas entre la producción gestual de las madres de ambos grupos ( $t$ $(28)=.77, p=.44)$; es decir, hay una producción similar de gestos deícticos en ambos grupos de díadas.

Ahora bien, respecto a la producción léxica, en los infantes de 30 meses de edad, se identificó una media de producción de palabras type de 42.93 ( $D E=24.03)$, así como una media de producción de palabras token de $113.80(D E=67.63)$. Para el caso de los infantes de 48 meses, hubo una media de producción de palabras type de $116.73(D E=29.28)$. Respecto a las palabras token, hubo una media de producción de 355.57 ( $D E=140.71$ ). Al comparar la producción de palabras type de ambos grupos de edad, se identificó que los infantes de 48 meses producen significativamente una mayor cantidad de palabras diferentes en comparación con los infantes de 30 meses $(t(28)=7.54, p=$ .000). Del mismo modo, se encontró diferencias significativas en la producción de palabras token de ambos grupos $(t(28)=6.00$, $p=.000)$ : los infantes de 48 meses emitieron una mayor cantidad de palabras -incluidas las palabras repetidas- en comparación con los infantes de 30 meses. Estos datos indican una tendencia en el aumento de la producción léxica conforme avanza la edad de los infantes.

El objetivo central de este estudio fue saber si existe una relación entre los gestos deícticos que usan las madres en interacción con sus hijos y la producción de palabras de los infantes en una tarea de lectura conjunta. El análisis de correlación con la prueba de Pearson arrojó los resultados que se presentan en la Tabla 1. 
Tabla 1. Correlaciones entre la producción de gestos deícticos y la producción léxica de los infantes (palabras type y palabras token).

Producción gestual de las madres
Producción léxica de los infantes

Palabras type

.279

Palabras token

Infantes- 30 meses

$.530 *$

.238

Infantes- 48 meses

Gestos deícticos

La correlación es significativa a nivel *0.05

Los datos de las díadas cuyos infantes son de 48 meses de edad indican que existe una correlación significativa entre la producción de gestos deícticos de la madre y la producción de palabras type, aunque no se encontró dicha relación entre los gestos deícticos y la producción de palabras token. En otras palabras, se identificó que a mayor cantidad de gestos deícticos producidos por las madres de las díadas de infantes de 48 meses, mayor será la producción de diferentes palabras por parte de sus hijos en esta tarea de lectura conjunta. Por otra parte, los resultados muestran que no hay relación entre la producción de deícticos de la madre y la producción léxica en los infantes de 30 meses de edad.

\section{Discusión y conclusiones}

El gesto puede estar asociado de dos maneras con el desarrollo del lenguaje: directamente, cuando la propia gesticulación del niño le ayuda a dar el siguiente paso en el aprendizaje porque reduce la carga cognitiva (véase Capone y McGregor, 2004, y Goldin-Meadow, 2014), o bien indirectamente, a través de la observación de la gestualidad de los padres y los cuidadores (Rowe y Goldin-Meadow, 2009; Stanfield, Williamson y Özçalişkan, 2013). Este estudio se centró en identificar la relación entre la gestualidad que producen las madres en interacción con sus hijos y el lenguaje productivo de los infantes en una tarea de lectura conjunta. 
Para el caso de las díadas con infantes de 30 meses, los resultados mostraron que no hay una relación entre el uso de gestos deícticos por parte de las madres y la producción léxica de los infantes. Este hallazgo concuerda con el obtenido por Iverson et al. (1999), quienes no encontraron relación entre la producción de gestos de señalar (un subtipo de gesto deíctico) de las madres y la producción de palabras de infantes italianos de 20 meses de edad. No obstante, es posible que en estas edades la relación entre gesto y lenguaje verbal se dé directamente y no a través de la observación de los gestos de las madres. De acuerdo con la evidencia de otros estudios, se asume la posibilidad de que a los 30 meses de edad el gesto tenga un efecto en su desarrollo lingüístico si el propio infante lo produce (Goodwyn, Acredolo y Brown, 2000; Rowe, Özçalişkan y Goldin-Meadow, 2008; Colonnesi, Stams, Koster y Noom, 2010). En este sentido, valdría la pena analizar si la gestualidad de las madres está asociada a la producción gestual de los infantes para, posteriormente, identificar si esta última, a su vez, está relacionada con su producción léxica.

Se ha identificado una correlación positiva entre la producción léxica de infantes de 30 meses y la producción léxica de sus madres (Valdés, 2015); es altamente probable que en este periodo del desarrollo, en el que emerge el fenómeno de la explosión de vocabulario en niños mexicanos y el desarrollo simbólico tiende a focalizarse en el uso de palabras (Galván, 2007; Hernández-Padilla, 2007), el estímulo gestual deíctico de las madres no se relacione con la producción de palabras de los infantes a razón de que su naturaleza simbólica es de otra índole.

Ahora bien, respecto a las díadas con infantes de 48 meses, la producción de gestos deícticos por parte de las madres sí estuvo asociada a la amplitud del vocabulario de los infantes; es decir, entre más gestos deícticos produjeron las madres, mayor fue la producción de palabras distintas por parte de sus hijos. En esta edad, entonces, los gestos deícticos cumplen con un papel relevante en el lenguaje productivo de los infantes en esta tarea de lectura conjunta. De acuerdo con los resultados, esta relación no se presentó con la producción de palabras token; esto indica que el uso de los gestos por parte de las madres no está asocia- 
do con cuánto habla el infante en interacción con su madre, pero sí con el número de palabras distintas que emplea. Así, el gesto posee una relación con la riqueza del vocabulario productivo.

Las madres y sus hijos, quienes interactúan en atención conjunta hacia un libro -especialmente la madre-, ponen en marcha estrategias comunicativas que fomentan el desarrollo del lenguaje (Ard y Beverly, 2004; Farrant y Zubrick, 2011). Gracias a este estudio se puede afirmar que los gestos deícticos también forman parte de dichas estrategias comunicativas. Dado que los gestos son un recurso para dirigir la atención del niño (De Villiers y Zukow-Goldring, 2010; Migdalek y Rosemberg, 2012), es probable que los gestos deícticos hayan cumplido justamente dicha función en la lectura conjunta, tarea en la que se requieren habilidades cognitivas como la atención.

Los datos de este estudio indican que, debido al propio desarrollo, entre más edad posee el infante aumenta su capacidad de habla. ¿Qué factores podrían estar relacionados con el desarrollo de su producción léxica? Como observamos en los resultados, aunque el proceder gestual de las madres es similar en ambas edades -no hubo diferencias estadísticamente significativas en la producción de gestos entre las madres de ambos grupos-; el uso del gesto deíctico estuvo asociado con la amplitud del vocabulario productivo de los infantes de 48 meses. ¿Por qué esta relación se identificó en infantes de 48 meses y no a los 30 meses? Es posible que este vínculo esté relacionado con su capacidad para comprender el gesto y extraer de este tipo de representación simbólica el significado que los adultos desean transmitir. En las siguientes líneas se discutirá esta afirmación.

El uso del gesto -producido por los padres- garantiza en los niños una mayor comprensión de los mensajes (Cook y Goldin-Meadow, 2006). Así, cuando se construyen mensajes a través del uso de gesto y habla -en el que el gesto refuerza el mensaje oral o agrega información al mismo (Greenfield y Smith, 1976; Özçalişkan y Goldin-Meadow, 2005)-, los niños de 4 años de edad tienden a presentar mayores habilidades para comprender los mensajes de sus padres, especialmente para extraer de los gestos información que no se expresa por medios orales -en comparación con los niños de 2 y 3 años (Stanfield, Williamson 
y Özçalişkan, 2013). Dado que los niños de 4 años tienen mayor capacidad para interpretar el gesto, es posible que eso les permita aprehender más elementos significativos. A pesar de que con los resultados de esta investigación no es posible afirmar que exista una relación causal entre las variables, se puede inferir en términos teóricos que el proceder narrativo de la madre con el uso de gestos deícticos permitió que los infantes de 48 meses, quienes poseen mayores habilidades de comprensión del gesto que emerge en combinación con el habla, se hicieran de más información para construir su propia narración y, por lo tanto, ostentar una mayor amplitud léxica. Esta podría ser una explicación viable sobre por qué la relación entre la producción gestual de las madres y la producción léxica de los infantes se observa en niños de 48 meses y no en niños de 30 meses de edad.

Aunque el alcance de esta investigación únicamente permite conocer la naturaleza de una producción léxica situada y limitada por una tarea específica, la lectura conjunta fue idónea para observar y medir las variables de interés. El diseño metodológico ha dado paso a un mayor control de la actividad comunicativa de los participantes: se centra en la construcción de un relato que versa sobre una serie de ilustraciones. El tipo de interacción que se suscitó entre los participantes es adecuado para estudiar tanto la producción gestual -tradicionalmente la gestualidad se ha estudiado en discursos narrativos- (McNeill, 1992) como la producción verbal en infantes (Ard y Beverly, 2004).

La tarea de lectura conjunta implica una dinámica de construcción de significado. Dicho significado se entreteje como resultado de una construcción social (Vigotsky, 1932 y 1934) en la que diferentes elementos simbólicos contribuyen en la representación y están asociados entre sí. En la construcción de un relato intervienen procesos intersubjetivos (Sankey-García y Gutiérrez-Estupiñán, 2006) en los que el gesto participa como una forma de significar; esta investigación ha mostrado que el proceder gestual de las madres está ligado a las acciones lingüísticas que los infantes emplean en su propia construcción de la narración. Aunque cada elemento de la díada tiene su turno para construir su discurso narrativo, es evidente que sus elementos representacionales están relacionados. En este sentido, los gestos también son 
elementos que participan en la configuración del significado (Minto-García, 2014), en el proceso intersubjetivo de construir un discurso que, si bien pertenece a la producción individual, es resultado de un proceso de construcción conjunta entre madres e hijos.

Aunque con este estudio no es posible proporcionar explicaciones concluyentes sobre el papel del gesto en el desarrollo de la producción léxica, los resultados permiten afirmar que el gesto está asociado con la riqueza de la producción léxica de los infantes. Este estudio ha proporcionado evidencia de que la gestualidad de las madres es un elemento asociado a la construcción de la narración de los infantes, específicamente a su producción de distintas palabras (palabras type). Estos hallazgos sugieren la importancia de continuar con el estudio del uso del gesto con miras a identificar su influencia en el desarrollo del lenguaje de los infantes, especialmente el tamaño del vocabulario productivo.

\section{Referencias}

Acredolo, L. y Goodwyn, S. (1988). Symbolic gesturing in normal infants. Child Development, 59, 450-466.

Alva, E. (2004). Modelos de desarrollo del lenguaje espontáneo en infantes y escolares: análisis de muestras masivas. Tesis de doctorado. Universidad Nacional Autónoma de México.

Anguera, M. T. (1983). Manual de prácticas de observación. México: Trillas.

Ard, L. M. y Beverly, B. L. (2004). Preschool word learning during joint book reading. Effect of adult questions and comments. Communication Disorders Quarterly, 26(1), 17-28.

Arias, N. (2014). La lectura conjunta y la interacción entre adultos y niños de 3-5 años. Revisión de antecedentes. Panorama, 8(14), 34-46. Recuperado de https://journal.poligran. edu.co/index.php/panorama/article/viewFile/503/453

Bavelas, J. B. y Chovil, N. (2006). Nonverbal and verbal communication: Hand gestures and facial displays as part of language use in face-to-face in dialogue. En Manusov, V. y Patterson, M. L. (Eds.), The Sage Handbook of Nonverbal Communication (pp. 97-115). Thousand Oaks, CA: Sage.

Bavelas, J. B., Kenwood, C., Johnson, T. y Phillips, B. (2002). An experimental study of when and how speakers use gestures to com- 
municate. Gesture, 2(1), 1-18.

Capirci, O., Iverson, J. M., Pizzuto, E. y Volterra, V. (1996). Gestures and words during the transition to two word speech. Journal of Child Language, 23, 645-673.

Capone, N. y McGregor, K. (2004). Gesture development: A review for clinical and research practices. Journal of Speech, Language and Hearing Research, 47, 173-186.

Capone, N. y Saks, J. (2015). Co-speech gesture input as a support for language learning in children with and without early language delay. Perspectives on Language Learning and Education, 22, 61-71.

Colonnesi, C., Stams, G. J. J. M., Koster, I. y Noom, M. J. (2010). The relation between pointing and language development: A meta-analysis. Developmental Review, 30, 352-366.

Cook, S. y Goldin-Meadow, S. (2006). The role of gesture in learning: Do children use their hands to change their minds? Journal of Cognition and Development, 7(2), 211-232.

De Ruiter, J. P., Bangerter, A. y Dings, P. (2012). The interplay between gesture and speech in the production of referring expression: Investigating the Tradeoff hypothesis. Topics in Cognitive Science, 4, 232-248.

De Villiers, N. y Zukow-Goldring, P. (2010). How the hands control attention during early word learning. Gesture, 10 (2-3), 202-221.

Farkas, C. (2007). Comunicación gestual en la infancia temprana: Una revisión de su desarrollo, relación con el lenguaje e implicancias de su intervención. Psykhe, 16(2), 107-115. Recuperado de https://scielo.conicyt.cl/pdf/psykhe/v16n2/art09.pdf

Farrant, B. M. y Zubrick, S. R. (2011). Early vocabulary development: The importance of joint attention and parent-child book reading. First Language, 32(3), 343-364.

Galván, A. (2007). Análisis de la explosión de vocabulario. En E. Alva (Ed.), Del universo de los sonidos a la palabra: Investigaciones sobre el desarrollo del lenguaje en infantes. México: UNAM.

Goldin-Meadow, S. (2000). Beyond words: The importance of gesture to researches and learners. Child Development, 71(1), 231-239. . (2009). How gesture promotes learning throughout childhood. Child Development Perspectives, 3(2), 106-111.

. (2014). Widening the lens: What the manual modality reveals about language, learning and cognition. Philosophical Transactions of the Royal Society of London: Series B. Biological Sciences, 369. 
. (2015). Gesture as a window onto communicative abilities: Implications for diagnosis and intervention. Perspectives on Language Learning and Education, 22(2), 50-60.

Goodwyn, S., Acredolo, L. y Brown, C. (2000). Impact of symbolic gesturing on early language development. Journal of Nonverbal Behavior, 24, 81-103.

Greenfield, P. y Smith, J. (1976). The structure of communication in early language development. New York: Academic Press.

Guerrero, B. V. (2016). Conductas parentales y desarrollo de habilidades lingüísticas. Tesis de doctorado. Universidad Nacional Autónoma de México.

Haviland, J. (2000). Pointing, gesture spaces, and mental maps. En McNeill, D. (Ed.), Language, Culture and Cognition 2. Language and Gesture (pp. 13-46). New York: Cambridge University Press. Hernández-Padilla, E. (2007). Categorías lexicales y la explosión del vocabulario. En E. Alva (Ed.), Del universo de los sonidos a la palabra: Investigaciones sobre el desarrollo del lenguaje en infantes. México: UNAM.

Iverson, J. M., Capirci, O., Longobardi, E. y Caselli, C. (1999). Gesturing in mother-child interactions. Cognitive Development, 14(1), 57-75. Jones, C. P. y Adamson, L. B. (1987). Language use in mother-child and mother-child-sibling interactions. Child Development, 58(2),356-366.

Kendon, A. (2007). On the origins of modern gesture studies. En Duncan, S. D., Cassell, J. y Levy, E. T. (Eds.), Gesture and the Dynamic Dimension of Language (pp. 13-28). Amsterdam, Philadelphia: John Benjamins Publishing Company.

Krauss, R. M., Chen, Y. y Gottesman, R. F. (2000). Lexical gestures and lexical access: A process model. En McNeill, D. (Ed.), Language, Culture and Cognition 2. Language and Gesture (pp. 261283). New York: Cambridge University Press.

Kuhn, L. J. et al. (2014). Early communicative gestures prospectively predict language development and executive function in early childhood. Child Development, 85(5), 1898-1914.

. (2016). The contribution of children's time-specific and longitudinal expressive language skills on developmental trajectories of executive function. Journal of Experimental Child Psychology, 148, 20-34.

Lewis, C. y Gregory, S. (1987). Parents' talk to their infants: the impor- 
tance of context. First Language, 7, 201-216.

Mayer, M. (1967). A boy, a dog, and a frog. New York: Dial Books for Young Readers.

. (1973). Frog on his own. New York: Dial Books for Young Readers.

McNeill, D. (1985). So you think gestures are nonverbal? Psychological Review, 92, 350-371.

McNeill, D. (1992). Hand and Mind. Chicago: University of Chicago Press.

Migdalek, M. J. y Rosemberg, C. (2012). El uso de los gestos en el discurso docente durante la planificación del juego en el jardín de infantes. Bellaterra Journal of Teaching and Learning Language and Literature, 5(3), 25-43. Recuperado de https://ddd.uab.cat/ pub/jtl3/jtl3_a2012m8-9v5n3/jtl3_a2012m8-9v5n3p25.pdf

Minto-García, A. (2014). Gestualidad y significado. Tesis de doctorado. Benemérita Universidad Autónoma de Puebla.

O’Neill, D. y Holmes, A. C. (2002). Young preschoolers' ability to reference story characters: the contribution of gestures and character speech. First Language, 22, 73-103.

Özçalişkan, S. y Dimitrova, N. (2013). How gesture input provides a helping hand to language development. Seminars in Speech and Language, 34(4), 227-236.

Özçalişkan, Ş. y Goldin-Meadow, S. (2005). Do parents lead their children by the hand? Journal of Child Language, 32(3), 481-505.

Özçalişkan, Ş., Genter, D. y Goldin-Meadow, S. (2014). Do iconic gestures pave the way for children's early verbs? Applied Psycholinguistics, 35(6), 1143-1162.

Pan, B. A., Rowe, M. L., Singer, J. D. y Snow, C. E. (2005). Maternal correlates of growth in toddler vocabulary production in low-income families. Child Development, 76(4), 763-782.

Romero, S. y Gómez, G. E. (2013). El desarrollo del lenguaje evaluativo en narraciones de niños mexicanos de 3 a 12 años. Actualidades en Psicología, 27(115), 15-30.

Rowe, M. L. y Goldin-Meadow, S. (2009). Differences in early gesture explain SES disparities in child vocabulary size at school entry. Science, 323, 951-953.

Rowe, M., Özçalışkan, S. y Goldin-Meadow, S. (2008). Learning words by hand: Gesture's role in predicting vocabulary development. 
First Language, 28(2), 185-203.

Sankey-García, M. R. y Gutiérrez-Estupiñán, R. (2006). El texto narrativo intersubjetivo. México: BUAP.

Stanfield, C. Williamson, R. y Özçalişkan, Ş. (2013). How early do children understand gesture-speech combinations with iconic gestures? Journal of Child Language, 1-10.

Suárez, P., Alva, E. A. y Valdés, T. (2016). Análisis de la complejidad del vocabulario en infantes de 36 meses de edad. Investigación y Práctica en Psicología del Desarrollo, 2, 8-23.

Vygotsky, L. (1932). El desarrollo de los procesos psicológicos superiores. México: Grijalbo. . (1934). Pensamiento y lenguaje. Barcelona: Paidós.

Zukow-Goldring, P. (1996). Sensitive caregiving fosters the comprehension of speech: when gestures speak louder than words. Early Development \& Parenting, 5, 195-211. 